\title{
Stafne's Defect: Diagnosis with Cone Beam Computed Tomography: Case Report
}

\author{
Gisele Pavão Spaulonci ${ }^{1 *}$, Emerson Eli Nunes Cunha², Luciano Lauria Dib ${ }^{3}$, Elcio Magdalena \\ Giovani $^{4}$ \\ ${ }^{1}$ Dentist - Master's Degree in Dentistry at the University Paulista, São Paulo, SP, Brazil \\ ${ }^{2}$ Radiology Technician - Radiology Departmentat the University Paulista, São Paulo, SP, Brazil. \\ ${ }^{3}$ Dentist - Teacher Titular Doctor of Stomatology and Postgraduate Course - Master and Doctorate in Dentistry at the University Paulista, São Paulo, \\ SP, Brazil. \\ ${ }^{4}$ Dentist - Teacher Titular Doctor of Integrated Clinic and Patients with Special Needs and the Postgraduate Course - Master and Doctorate in \\ Dentistry at the University Paulista, São Paulo, SP, Brazil.
}

Received: December 12, 2016; Accepted: December 27, 2016; Published: January 52017

*Corresponding author: Gisele Pavão Spaulonci,Master's Degree in Dentistry at the University Paulista, Dr. Bacelar Street, 1212 , São Paulo - SP, Brazil, CEP: 04026-002,TEL:55 (11) 98772-7772;Fax: 55 (11) 3801-4011;E-mail: giselepavao@terra.com.br

\begin{abstract}
Stafne's Defect is an asymptomatic bone lesion, most common in men between the fifth and seventh decade of life. It is characterized as radiolucent, delimited and well-defined image in the posterior region of the mandible and is usually discovered on routine radiographic examination. In this case, the patient is in the third decade of life and clinical and radiographic characteristics described were consistent with those found in academic literature. Cone beam computed tomography was used to diagnose the defect and enabled the differential diagnosis with other pathologies, avoiding unnecessary surgical procedures. Considering that it is a non-progressive change, its conservative therapy is based on the periodic control of the lesion.

In this case report, it was possible to confirm that cone beam computed tomography acts as an important tool in the diagnosis of Stafne'sDefect, as it enabled the differential diagnosis in regards to other diseases, thus avoiding unnecessary surgical procedures.
\end{abstract}

Keywords: Stafne's defect; Cone beam computed tomography; Diagnosis;

\section{Introduction}

Stafne'sBone Defect is an asymptomatic bone lesion, most common in men between the fifth and seventh decade of life. It is often diagnosed in routine radiographs (1-3). There are two variations of Stafne'sDefect, a posterior and anterior variants(2). The posterior variation is more commonly known and appears as radiolucent, delimited and well-defined image in the posterior region of the mandible, between the first molar and the angle of the mandible, below the mandibular canal $(2,4)$. The anterior variant is 7 times less frequent than the posterior, and usually located in the region between the incisors and premolars, above the insertion of the mylohyoid muscle $(5,6)$.

Its etiology is still uncertain, however it is more commonly accepted that it is caused by pressure from the submandibular gland, generating reabsorption in the lingual aspect of the mandible, thus causing the injury $(3,5)$. The submandibular gland is related to the posterior variant of Stafne'sDefect,the sublingual gland may be related to the anterior variant while the parotid gland is related with the two types of Stafne's Defect of the mandibular ramus $(1,5)$. This etiology is most commonly accepted and is supported by the patients 'age (4), and there are findings in which the patients had taken previous radiographs that showed no injuries. Due to controversies regarding its etiology, this injury has been called many names, including cavity or static bone defect, idiopathic bone cavity or latent bone cavity, ectopic submandibular and sublingual gland in the mandible, mandibular lingual cortical defect, defect or cavity of Stafne, and Stafne cyst $(4,7,8)$.

In the present study, we describe a case of Stafne'sDefect diagnosed in radiological findings using cone beam computed tomography as a means for the diagnostic.

\section{Case Report}

A 39-year-old male patient, leucoderma arrived for a checkup dental visit and complained about discomfort in the region of the tooth 18 , which had clinically residual root. After referring the patient to the radiology department for panoramic radiography, a radiolucent, limited and well-defined image was found in the posterior region of the mandible, on the left side near the root of tooth 38. During the clinical assessment, the patient confirmed not having any symptoms on the corresponding area. It was requested a Cone Beam Computed Tomography for diagnostic confirmation of Stafne's Bone Defect.

For diagnosis confirmation, we have used Cone Beam Volumetric Computed Tomography with Sirona Orthophos XG3D equipment, operating at $70 \mathrm{KV}, 10 \mathrm{MA}$ and $14.2 \mathrm{~s}$ of exposure time for image acquisition.

Through panoramic view, a radiolucent, limited and welldefined image was found in the jaw structure, on the left side as shown in (Figure 1). 
During the axial reconstruction, a lesion was observed on the lingual cortical of the mandible, as shown in( Figure 2). The coronal section showed a lesion measuring $6.38 \mathrm{~mm}$ in the central region and $6.86 \mathrm{~mm}$ on the lingual edge, as shown in (Figure 3).

In the $3 \mathrm{D}$ reconstruction, there was a radiolucent image in the posterior region of the mandible, on the left side (Figure 4), and the Tangential cross-section allowed us to verify the circumscribed lesion, well outlined by hyper dense halo, measuring $13.15 \mathrm{~mm}$ as in (Figure 5).

\section{Discussion}

Stafne'sBone Defect is an asymptomatic bone lesion, most common in men between the fifth and seventh decade of life. It is often diagnosed in routine radiographs (1-3), similar to what was observed in this case. However, patient age was different to that reported in academic literature, as the patient in question was at the end of his third decade of life upon diagnosis. In a case published by Lee et al. (8), a case of Stafne'sBone Defect was also reported in a patient with 30 years of age. Münevveroğlu and Aydin (9) published a case of a patient with Stafne'sDefect diagnosed at age 31 .

The posterior variation of the injury is more common and usually presents itself as a radiolucent, delimited and well-defined image between the angle of the mandible and the first molar, below the mandibular canal which was similar to the present

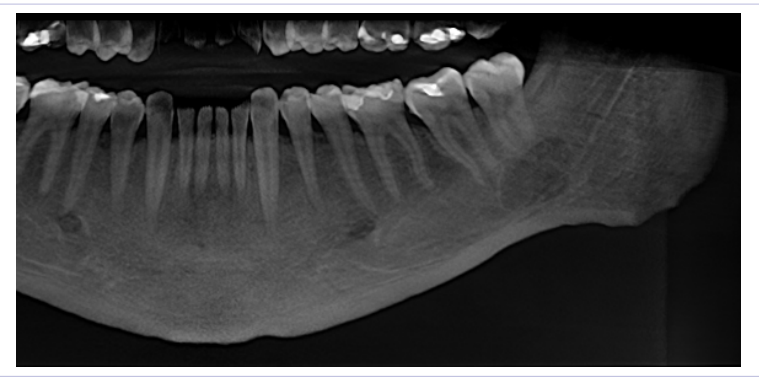

Figure 1: Radiolucent, delimited and well-defined image, located on the left side in theposterior region of the mandible, as seen in Cone Beam Computed Tomography - Panoramic View.

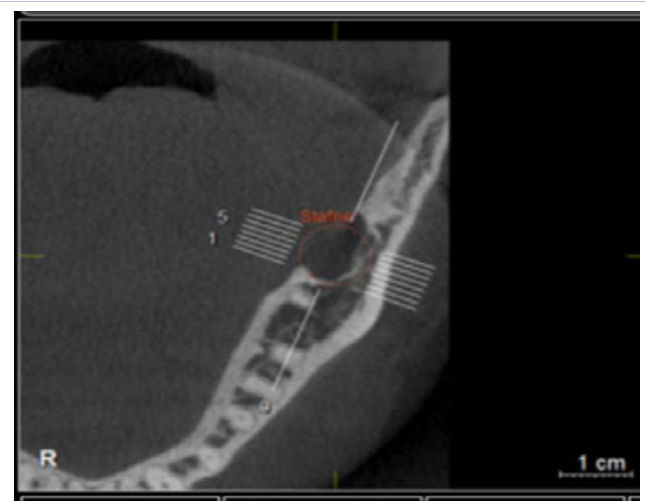

Figure 2: Radiolucent image in mandibular lingual cortical observed in Cone Beam Computed Tomography - Axial Reconstruction.

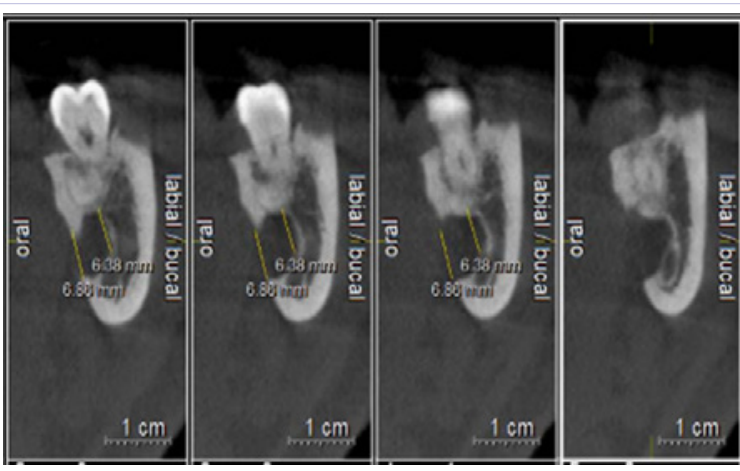

Figure 3: Radiolucent image in the mandibular lingual cortical, measuring $6.38 \mathrm{~mm}$ in the central region and $6.86 \mathrm{~mm}$ in the lingual edge, as seen in Cone Beam Computed Tomography, coronal section.

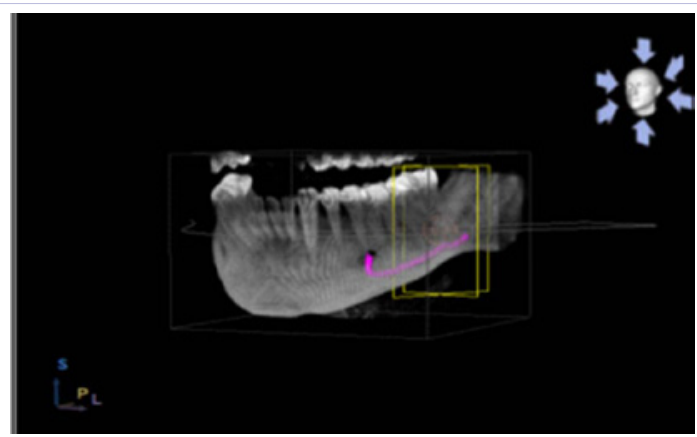

Figure 4: Radiolucent, delimited and well-defined image located on the left side in the posterior region of the mandible, as seen in Cone Beam Computed Tomography - 3D reconstruction.

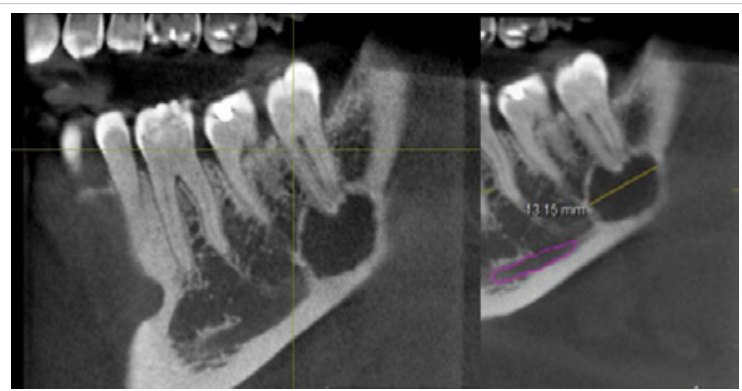

Figure 5: Radiolucent, delimited and well-defined image by hyperdense halo, measuring $13.15 \mathrm{~mm}$ on the left side in the posterior region of the mandible, as seen in Cone Beam Computed Tomography - Tangential Cross-Section.

case. However, there are reports of lesions in the anterior region $(2,10)$. Dereci et al. (2) published a case of anterior Stafne's Bone Defect with intraoral exposure.

Lesions occur on the lingual surface of the mandible, as shown in this case. However, Herranz-Aparicio et al. (1) found an unusual case involving the lingual and buccalmandibularplates in which the examination by computed tomography showed a tunnel-like lesion with a complete loss of continuity of both lingual and buccalplates. 
Between the two types of defect variations, the anterior is rarer and more difficult to diagnose compared to the posterior variant. According to their radiographic characteristics, there are some diseases that present differential diagnosis, as odontogenic or non-odontogenic cystic lesions, fibrous dysplasia, non-ossifying fibroma, brown tumor of hyperparathyroidism, ameloblastoma, giant cell tumor, benign salivary gland tumors, hemangioma, myxoma, multiple myeloma, eosinophilic granuloma and metastatic disease (7).

According to Ávila et al. (2008), imaging tests are essential for the differential diagnosis because they provide valuable information on the hard and soft tissues without the need for exploratory surgical procedures (3).

In current days, computed tomography is considered the complementary test of choice to aid in the diagnosis, as it has great advantage in the checking of the peripheral origin of the lesion and the visualization of the concavity in the lingual cortical, which are essential features to make the differential diagnosis with other pathologies (5).

In this case, we have used cone beam computed tomography with Sirona Orthophos XG3D equipment, operating at $70 \mathrm{KV}$, $10 \mathrm{MA}$ and $14.2 \mathrm{~s}$ of exposure time to support the diagnosis. The lingual concavity was identified through axial and coronal cross-sections (Figures 2 and 3), in addition to other clinical and radiographic characteristics, there was consonance of this case with those found in academic literature.

Other studies have also used the cone beam computed tomography to aid in the diagnosis of Stafne's Bone Defect $(3,4$, $6,9)$.

Magnetic resonance imaging can be used in cases where computed tomography or conventional radiography are unable to provide a correct diagnosis, taking advantage of the precise identification of the soft tissue (4).

Sialography can also be considered. It is a radiographic examination using contrast media inside the duct of the glands. However, this is an invasive procedure that may cause discomfort to the patient, and can be contraindicated for the opportune diagnosis of the anterior variant of Stafne's Defect due to numerous Rivini ducts, which have smaller diameter and may suffer rupture during the procedure $(1,4)$.

The use of correct imaging examinations is of greatimportance in order to avoid unnecessary invasive procedures (4), since conservative therapy is based on the periodic control of the injury. Currently, complementary techniques such as computed tomography are sufficient to establish the correct diagnosis (9).

\section{Conclusion}

In this case report it was possible to determine that Cone Beam Computed Tomography is an important tool for the diagnosis of Stafne's Bone Defect, given that it allowed the visualization of the lingual bone concavity in the posterior region of the mandible, and enabled the differential diagnosis with other diseases when combined with other clinical and radiographic characteristics, thus avoiding unnecessary surgical procedures or invasive procedures such as Sialography.

\section{References}

1. Herranz-Aparicio J, Figueiredo R, Gay-Escoda C. Stafne's bone cavity: An unusual case with involvement of the buccal and lingual mandibular plates. J clinexp Dent.2014;6(1):e96-99. doi: 10.4317/ jced.51229.

2. DereciO,Duran S. Intraorally exposed anterior Stafne bone defect: a case report. Oral Surg Oral Med Oral Pathol Oral Radiol.2012;113(5):e1-3. doi: 10.1016/j.tripleo.2011.07.029.

3. Ávila LD, Dias-Ribeiro E, Sampieri MBS, Ferreira-Júnior O, Santa'ana E. Cistoósseo de Stafne - árearadiolúcidanamandíbula versus patologiasósseas: revisão de literatura | Rev. Cir.traumatol. Bucomaxilo-fac;9(3)jul.-set. 2009. Ilus | LILACS | Portal Regional da BVS [Internet]. [citado 4 de setembro de 2016]. Disponívelem: http:// pesquisa.bvsalud.org/portal/resource/pt/lil-535404

4. Villoria EM, Cunha CHR da, Francio LA, Noia J, Taitson PF, Spyrides KS, et al. Diagnóstico do defeitoósseo de Stafne pormeio de tomografiacomputadorizada de feixecônico: relato de caso. Rev faculdadeodontollins.2013;23(1):53-58. doi.org/10.15600/22381236/fol.v23n1p53-58.

5. Quesada-Gómez C, Valmaseda-Castellón E, Berini-Aytés L, Gay-Escoda C. Stafne bone cavity: a retrospective study of 11 cases. Med Oral Patol Oral Cir Bucal. 2006;11(3):E277-280.

6. Sisman Y, Miloglu O, Sekerci AE, Yilmaz AB, Demirtas O, Tokmak TT. Radiographic evaluation on prevalence of Stafne bone defect: a study from two centres in Turkey. Dentomaxillo Facial Radiol. 2012;41(2):152-158. doi.org/10.1259/dmfr/10586700.

7. Kaya M, Ugur KS, Dagli E, Kurtaran H, Gunduz M. Stafne bone cavity containing ectopic parotid gland. Braz J Otorhinolaryngol.2016. doi. org/10.1016/j.bjorl.2016.02.004

8. Lee KH, Thiruchelvam JK, mcdermott P. An Unusual Presentation of Stafne Bone Cyst. J Maxillofac Oral Surg. Setembro de .2015;14(3):841844. DOI: $10.1007 / \mathrm{s} 12663-014-0737-2$.

9. Münevveroğlu AP, Aydın KC. Stafne bone defect: report of two cases. Case Rep Dent. 2012;2012(2012):654839;5. doi. org/10.1155/2012/654839.

10. Griffa A, Zavattero E, Passalacqua F, Berrone S. Anterior Stafne bone defect mimicking an odontogenic cyst. J Craniofac Surg. 2014;25(3):1126-1128. DOI:10.1097/SCS.0000000000000539. 